Original Article

\title{
Effects of physical therapy on blood pressure in daily clinical practice-a pilot study
}

\author{
Shota Yamada, MS ${ }^{1)}$, Gen Nitta, BS ${ }^{2)}$, Yuki Takano, BS ${ }^{3)}$, Satomi Yoshimura, BS ${ }^{4)}$, \\ KaZUji AoKi, $\mathrm{MS}^{5)}$, YasuaKi Dohi, $\mathrm{MD}, \mathrm{PhD}^{5)^{*}}$ \\ 1) Physical Medicine and Rehabilitation, Nagoya City West Medical Center, Japan \\ 2) Department of Rehabilitation, Fujieda Heisei Memorial Hospital, Japan \\ 3) Department of Rehabilitation, Kashiwazaki General Hospital and Medical Center, Japan \\ 4) Department of Rehabilitation, Municipal Ena Hospital, Japan \\ 5) Faculty of Rehabilitation Sciences, Nagoya Gakuin University: 3-1-17 Taiho, Atsuta-ku, \\ Nagoya 456-0062, Japan
}

\begin{abstract}
Purpose] Most exercise therapy procedures induce hemodynamic changes and could be a cardiovascular risk. This pilot study investigated factors that induce an exaggerated increase in blood pressure during exercise therapy. [Participants and Methods] We measured the blood pressure and pulse rate before and after exercise therapy for ambulation on days 1,2 , and 7 of the exercise therapy in patients ( $n=23$; age, $69 \pm 11$ years) who were hospitalized for a stroke or an orthopedic surgery. [Results] Each participant's blood pressure and pulse rate were significantly increased after the exercise therapy. Regression analysis demonstrated that the increase in systolic blood pressure was independently predicted by body weight and pulse rate before the exercise therapy. In the logistic regression analysis, age and body weight were independent predictors of the exaggerated increase in systolic blood pressure (fourth quartile). [Conclusion] A significant increase in blood pressure was induced by exercise therapy. There was a correlation between systolic blood pressure increase and pulse rate before the exercise therapy. Old age or increased body weight predicts exaggerated increase in blood pressure during exercise therapy. Key words: Elevation of systolic blood pressure, Exercise therapy, Pulse rate
\end{abstract}

(This article was submitted Feb. 26, 2020, and was accepted Apr. 3, 2020)

\section{INTRODUCTION}

Hypertension, defined based on blood pressure measured in the office or at home in a resting condition, is an important risk factor for cardiovascular disease ${ }^{1}$. However, in response to various physical and mental stimuli, blood pressure shows marked diurnal or circadian fluctuations, and the clinical significance of such short-term or transient changes in blood pressure has not been fully understood ${ }^{2}$. It has been shown that blood pressure can increase during resistance training ${ }^{3-6)}$, which is much more pronounced in individuals with hypertension than in those without, but an abrupt increase in blood pressure may be a cardiovascular risk. Indeed, morning surge, which is an exaggerated increase in blood pressure early in the morning, is a strong predictor of stroke, coronary heart disease, and all-cause death in patients with hypertension ${ }^{7)}$ and cardiovascular events most frequently occur just after waking up, when blood pressure shows a rapid increase (as determined by ambulatory blood pressure monitoring $)^{8}$.

Physical therapy, especially most exercise therapy procedures, has an effect on hemodynamics ${ }^{4,9)}$ and thus could be a cardiovascular risk in some cases ${ }^{10}$. Therefore, the conditions of patients should be properly evaluated and hemodynamic indices, including blood pressure and heart rate, should be monitored to minimize cardiovascular risk during exercise therapy.

*Corresponding author. Yasuaki Dohi (E-mail: ydohi@ngu.ac.jp)

(C2020 The Society of Physical Therapy Science. Published by IPEC Inc.

(c) (1) $($ This is an open-access article distributed under the terms of the Creative Commons Attribution Non-Commercial No Derivatives

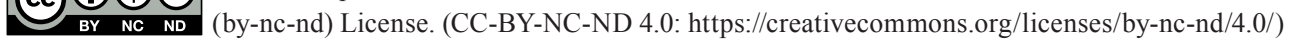


However, blood pressure is monitored only by $<25 \%$ physical therapists ${ }^{10)}$, probably because of incomplete evidence on establishing a normal or safety range for blood pressure response during exercise therapy. Although a stopping rule for physical therapy has been proposed by Anderson ${ }^{11)}$, there is no established evidence-based standard for stopping or continuing exercise therapy, which is attributable in part to the lack of data on blood pressure response to exercise therapy. Therefore, this pilot study was conducted to investigate how blood pressure is affected by exercise therapy in clinical practice.

\section{PARTICIPANTS AND METHODS}

This was a multicenter, pilot, observational study assessing the effects of exercise therapy on blood pressure in patients hospitalized for stroke or operation for an orthopedic disease. The study was performed in accordance with the principles of the Declaration of Helsinki, and the study design was approved by the ethics committee of Nagoya Gakuin University (2016-29), the core center of the study, and the local ethics committees of each hospital. Before participating in the study, all participants provided written informed consent. Data collection and statistical analyses were the responsibility of the core study center, and each investigator undertook recruitment and follow-up of patients. This study is registered with the UMIN Clinical Trial Registry (UMIN R000031958; http://www.umin.ac.jp/ctr).

This study included screened inpatients who underwent physical therapy from June 2017 to August 2018. Inclusion criteria included age $\geq 20$ years, being hospitalized in our hospitals for a stroke or an orthopedic surgery, and undergoing exercise therapy for getting up $(n=23)$. Patients in whom blood pressure could not be measured accurately because of arrhythmia were excluded. Stroke included ischemic and hemorrhagic stroke, and orthopedic surgery included spine surgery and the lower limb bone surgery, such as bipolar hip arthroplasty and total knee arthroplasty. Blood pressure and pulse rate were measured in a supine position after 2 min of rest at the start of exercise therapy and after the exercise therapy on days 1,2 , and 7 . The exercise therapy included range-of-motion exercise, strength training, training in standing up, and walking training. Medical records were used to obtain participant medical information. To assess pain during each exercise therapy, a $0-10$ numerical rating scale was used, where 0 indicates no pain and 10 indicates worst pain imaginable. An automated device (HEM-7130HP, Omron, Kyoto, Japan) was used to measure blood pressure.

Criteria for hypertension were as follows: systolic blood pressure $\geq 140 \mathrm{mmHg}$, diastolic blood pressure $\geq 90 \mathrm{mmHg}$ ), or antihypertensive medication use. The presence of diabetes mellitus was determined as a fasting plasma glucose level of $\geq 126 \mathrm{mg} / \mathrm{dL}^{12}$ ) or antidiabetic medication use, whereas dyslipidemia was defined as a low-density lipoprotein cholesterol level of $\geq 140 \mathrm{mg} / \mathrm{dL}$, a high-density lipoprotein cholesterol level of $<40 \mathrm{mg} / \mathrm{dL}$, a triglyceride level of $\geq 150 \mathrm{mg} / \mathrm{dL}^{13)}$, or antidyslipidemic medication use.

IBM SPSS statistics 26 (IBM SPSS, Chicago, IL, USA) was used to perform all analyses, and data are presented as mean $\pm \mathrm{SD}$ or as $n(\%)$ of participants. Student paired t-test was used to determine the significance of differences between blood pressure or pulse rate values measured before and after physical therapy. Subsequently, data obtained on days 1, 2, and 7 of exercise therapy from each participant were pooled and the following analyses were conducted. To investigate the factors associated with changes in systolic blood pressure after exercise therapy, simple and multiple regression analyses were performed. Pooled data were divided into quartiles according to the level of change in blood pressure after exercise therapy, where the fourth quartile was defined as an exaggerated increase in blood pressure induced by exercise therapy. Multiple logistic regression analysis was performed, with the endpoint being exaggerated systolic blood pressure increase after exercise therapy. In all cases, $\mathrm{p}<0.05$ (two tailed) was considered significant.

\section{RESULTS}

Table 1 details the characteristics of the participants enrolled in this study. Most of them were hospitalized for orthopedic surgery and more than half of them were with hypertension (Table 1). Both blood pressure and pulse rate increased after exercise therapy when the 3-day (days 1,2, and 7) mean values for each patient were analyzed. The increases in diastolic blood pressure were slightly smaller than increases in systolic blood pressure (Table 2). A consistent increase in systolic, but not diastolic, blood pressure after exercise therapy was indicated in the comparative analysis of blood pressure changes after exercise therapy on days 1,2, and 7 (Table 3). The greatest response in systolic blood pressure in this study was an increase of $40 \mathrm{mmHg}$ in one participant on the first day of physical therapy, although the increase in systolic blood pressure in this participant in the succeeding exercise therapies was $<40 \mathrm{mmHg}$. There was no change in either blood pressure or pulse rate with repeated exercise therapy (Table 4).

In the next series of analyses, we examined the factors predicting the increase in blood pressure after exercise therapy by using pooled data collected on days 1, 2, and 7 of exercise therapy in each patient. The regression analysis showed that pulse rate before exercise therapy (i.e., at rest) and the presence of diabetes mellitus independently predicted the amplitude of increase in systolic blood pressure after exercise therapy (Table 5, Model A). Other multivariate models where the number of independent variables included were reduced confirmed that pulse rate before the exercise therapy was the independent predictor of systolic blood pressure increase (Table 5, Models B, C, and D). In dividing the responses of systolic blood pressure into quartiles, the fourth quartile was defined as an exaggerated increase in systolic blood pressure. The logistic regression analysis showed that the independent predictors of the exaggerated increase in systolic blood pressure after exercise therapy were age and body weight (Table 6). 
Table 1. Characteristics of participants

\begin{tabular}{lc}
\hline Characteristic & Value \\
\hline Male & $12(52.2)$ \\
Age (years) & $69.2 \pm 11.4$ \\
Body height (cm) & $159.3 \pm 8.8$ \\
Body weight (kg) & $62.6 \pm 15.8$ \\
Systolic blood pressure (mmHg) & $126.5 \pm 13.4$ \\
Diastolic blood pressure (mmHg) & $80.5 \pm 10.8$ \\
Pulse rate (bpm) & $74.9 \pm 14.2$ \\
Pain scale a $^{\text {a }}$ & $4.1 \pm 2.5$ \\
Reason for hospitalization & $21(91.3)$ \\
$\quad$ Orthopedic surgery & $2(8.7)$ \\
$\quad$ Stroke & $14(60.9)[9(64.3)]$ \\
Comorbidities & $2(8.7)[2(100)]$ \\
$\quad$ Hypertension [under medication] & $7(30.4)[6(85.7)]$ \\
$\quad$ Diabetes mellitus [under medication] & \\
$\quad$ Dyslipidemia [under medication] & \\
\hline Data are presented as mean \pm SD or $n$ (\%) of participants. The numbers in [] indicate \\
the number and percentage of patients under medication. \\
a Pain was assessed using a $0-10$ numerical rating scale, where 0 is no pain and 10 is \\
the worst pain imaginable.
\end{tabular}

Table 2. Responses of blood pressure and pulse rate after exercise therapy for getting up in patients hospitalized for a stroke or orthopedic operation

\begin{tabular}{lccc}
\hline & Before exercise therapy & After exercise therapy & p value \\
\hline Systolic blood pressure $(\mathrm{mmHg})$ & $127.7 \pm 11.4$ & $139.1 \pm 15.5$ & $<0.001$ \\
Diastolic blood pressure $(\mathrm{mmHg})$ & $80.9 \pm 8.8$ & $84.0 \pm 10.4$ & 0.006 \\
Pulse rate $(\mathrm{bpm})$ & $73.2 \pm 11.2$ & $76.5 \pm 14.6$ & 0.004 \\
\hline
\end{tabular}

The mean value of 3 days (days 1, 2, and 7) for each patient was taken as the response in each patient.

Table 3. Blood pressure and pulse rate measured before and after exercise therapy for getting up on days 1,2 , and 7 in patients hospitalized for a stroke or an orthopedic operation

\begin{tabular}{lrrr}
\hline & Before exercise therapy & After exercise therapy & p value \\
\hline Day 1 & & & \\
Systolic blood pressure (mmHg) & $126.5 \pm 13.4$ & $139.3 \pm 18.0$ & $<0.001$ \\
Diastolic blood pressure (mmHg) & $80.5 \pm 10.8$ & $86.1 \pm 12.3$ & $<0.001$ \\
Pulse rate (bpm) & $74.9 \pm 14.2$ & $80.3 \pm 18.7$ & 0.002 \\
Day 2 & $128.0 \pm 15.8$ & $139.0 \pm 19.0$ & $<0.001$ \\
Systolic blood pressure (mmHg) & $82.4 \pm 10.9$ & $84.2 \pm 12.7$ & 0.12 \\
Diastolic blood pressure (mmHg) & $72.5 \pm 12.2$ & $77.0 \pm 17.5$ & 0.006 \\
Pulse rate (bpm) & & & $<0.001$ \\
Day 7 & $123.6 \pm 13.8$ & $139.9 \pm 18.0$ & $<0.001$ \\
Systolic blood pressure (mmHg) & $78.4 \pm 10.9$ & $83.7 \pm 10.2$ & 0.62 \\
Diastolic blood pressure (mmHg) & $74.3 \pm 14.9$ & $75.0 \pm 15.2$ & \\
Pulse rate (bpm) & & & \\
\hline
\end{tabular}

Table 4. Changes in blood pressure and pulse rate after exercise therapy for getting up on days 1, 2, and 7 in patients hospitalized for a stroke or an orthopedic operation

\begin{tabular}{lccc}
\hline & Day 1 & Day 2 & Day 7 \\
\hline Changes in & & & \\
Systolic blood pressure (mmHg) & $12.7 \pm 13.1$ & $11.0 \pm 10.2$ & $16.3 \pm 9.7$ \\
Diastolic blood pressure (mmHg) & $5.6 \pm 6.2$ & $1.8 \pm 5.2$ & $5.3 \pm 4.3$ \\
Pulse rate (bpm) & $11.2 \pm 17.3$ & $11.7 \pm 17.4$ & $9.4 \pm 17.5$ \\
\hline
\end{tabular}

Response to exercise did not change throughout the observation period (ANOVA). 
Table 5. Results of regression analysis showing factors associated with systolic blood pressure response to exercise therapy

\begin{tabular}{lrc}
\hline & Standardized $\beta$ & p value \\
\hline Simple regression & & \\
Age (years) & 0.136 & 0.27 \\
Gender (male) & -0.047 & 0.70 \\
Body weight (kg) & -0.031 & 0.81 \\
Body height (cm) & -0.055 & 0.66 \\
Systolic blood pressure (mmHg) & -0.008 & 0.95 \\
Diastolic blood pressure (mmHg) & 0.054 & 0.66 \\
Pulse rate (bpm) & 0.242 & 0.46 \\
Pain scale & 0.004 & 0.97 \\
Hypertension & 0.100 & 0.42 \\
Diabetes mellitus & -0.121 & 0.33 \\
Multiple regression (Model A) & & \\
Age (years) & 0.204 & 0.24 \\
Gender (male) & -0.047 & 0.77 \\
Body weight (kg) & 0.437 & 0.06 \\
Systolic blood pressure (mmHg) & -0.138 & 0.29 \\
Pulse rate (bpm) & 0.376 & 0.02 \\
Pain scale & 0.017 & 0.91 \\
Hypertension & 0.100 & 0.44 \\
Diabetes mellitus & -0.561 & 0.006 \\
Multiple regression (Model B) & & \\
Age (years) & 0.165 & 0.32 \\
Gender (male) & -0.001 & 0.99 \\
Body weight (kg) & 0.401 & 0.08 \\
Pulse rate (bpm) & 0.376 & 0.02 \\
Hypertension & 0.090 & 0.48 \\
Diabetes mellitus & 0.376 & 0.02 \\
Multiple regression (Model C) & & \\
Age (years) & 0.001 & 0.99 \\
Gender (male) & -0.117 & 0.36 \\
Pulse rate (bpm) & 0.329 & 0.03 \\
Hypertension & 0.060 & 0.64 \\
Diabetes mellitus & -0.292 & 0.04 \\
Multiple regression (Model D) & -0.022 & 0.88 \\
Age (years) & 0.328 & 0.03 \\
Pulse rate (bpm) & 0.067 & 0.06 \\
Hypertension & -0.262 & \\
Diabetes mellitus & & 50 \\
\hline Mod A inclus age & \\
\hline
\end{tabular}

Model A includes age, gender, body weight, SBP, pulse rate, pain scale, hypertension, and diabetes mellitus as independent variables. Model B includes variables that show $\mathrm{p}<0.90$ in simple regression analysis are included as independent variables. Model $\mathrm{C}$ includes variables that show $\mathrm{p}<0.80$ in simple regression analysis are included as independent variables. Model $\mathrm{D}$ includes variables that show $\mathrm{p}<0.50$ in simple regression analysis are included as independent variables all variables listed in simple regression analysis.

${ }^{\text {a }}$ Pain was assessed using a $0-10$ numerical rating scale, where 0 is no pain and 10 is the worst pain imaginable.

\section{DISCUSSION}

This study demonstrates the following: (1) an increase in blood pressure is induced by exercise therapy for getting up in patients who experience a stroke or those who undergo orthopedic surgery; (2) at least during the first week of the start of exercise therapy, the increase in blood pressure is not attenuated with repeated exercise therapy; (3) pulse rate can predict the increase in systolic blood pressure after exercise therapy; and (4) elderly or overweight patients have a high risk of exaggerated increase in blood pressure.

Physical stress, such as that experienced with physical training, causes hemodynamic changes to supply adequate muscle blood perfusion, consisting usually of systolic blood pressure and heart rate increases and a redistribution of blood ${ }^{3-6)}$. Exercise therapy for getting up may also induce an increase in blood pressure. It has been empirically accepted that there will be brief increases in blood pressure after exercise therapy for getting up, but there have been no investigations on the characteristics of the response of blood pressure to exercise therapy. Although such changes in hemodynamics are a physiological phenomenon, sudden increases in blood pressure may be a cardiovascular risk, even if they are brief, especially in individuals with cardiovascular diseases ${ }^{10}$. In this context, clinically useful information was provided in this study, in which modest, but not serious, increases in blood pressure (in most cases with $<40 \mathrm{mmHg}$ ) and pulse rate, which are generally regarded 
Table 6. Factors predicting exaggerated increase in systolic blood pressure after physical therapy

\begin{tabular}{lcc}
\hline & Odds ratio & p value \\
\hline Model A & & 0.04 \\
Age (years) & 1.123 & 0.45 \\
Gender (male) & 2.204 & 0.01 \\
Body weight (kg) & 1.212 & 0.45 \\
Systolic blood pressure (mmHg) & 0.976 & 0.39 \\
Pulse rate (bpm) & 0.972 & 0.39 \\
Pain scale & 1.200 & 0.39 \\
Hypertension & 0.388 & 0.99 \\
Diabetes mellitus & 0.998 & \\
Model B & & 0.04 \\
Age (years) & 1.093 & 0.66 \\
Gender (male) & 1.484 & 0.01 \\
Body weight (kg) & 1.176 & 0.57 \\
Systolic blood pressure (mmHg) & 0.983 & 0.56 \\
Hypertension & 0.651 & 0.99 \\
Diabetes mellitus & 0.999 & \\
Model C & & 0.04 \\
Age (years) & 1.084 & 0.77 \\
Gender (male) & 1.278 & 0.01 \\
Body weight (kg) & 1.169 & 0.49 \\
Hypertension & 0.606 & 0.99 \\
Diabetes mellitus & 0.999 & \\
\hline
\end{tabular}

The results of the logistic regression analysis with the endpoint of an exaggerated elevation of systolic blood pressure (fourth quartile) after exercise therapy are shown in this table. All independent variables included in each model are listed in the table.

a Pain was assessed using a $0-10$ numerical rating scale, where 0 is no pain and 10 is the worst pain imaginable.

as safe, were induced by exercise therapy for getting up (including range-of-motion exercise, strength training, training in standing up, and walking training). Often, pain is experienced during exercise therapy, which usually promotes an increase in blood pressure ${ }^{14)}$. However, in our study, pain as assessed by the numerical rating scale did not affect changes in systolic blood pressure. The reason for this is not clear. A limitation to the assessment of pain could be the use of the numerical rating scale, as objective assessment of levels of pain is not possible. Alternatively, factors other than pain, such as vasodilatation caused by exercise, might have a higher prominent impact on hemodynamic changes. Future studies are needed to clarify this point. In this study, the increase in diastolic blood pressure was not consistent, in contrast to the response of systolic blood pressure. In healthy individuals, diastolic blood pressure remains unchanged during low-intensity dynamic exercises ${ }^{9,15)}$, whereas diastolic blood pressure is increased in high-intensity exercises ${ }^{16-18)}$. Other investigators have reported that changes in diastolic blood pressure during exercise are correlated with serum cholesterol and insulin resistance ${ }^{19}$. Thus, the results may have been affected by the different combination of exercises used in the exercise therapy in different participants, the different days in which each participant performed the exercise therapy, or a heterogeneity of participant background.

There was an independent correlation between the increase in systolic blood pressure after exercise therapy and pulse rate before the start of exercise therapy. An increased pulse rate at rest may partially indicate increased sympathetic nerve activity. Participants with increased sympathetic nerve system activity may have shown increased response of blood pressure after the exercise therapy. Participants with increased pulse rate at rest should be carefully observed and their blood pressure should be frequently monitored during exercise therapy; these may be useful to avoid unexpected and dangerous blood pressure increases. The results of the linear regression analysis were taken on the assumption that there are linear relationships between the increase in systolic blood pressure after exercise therapy and the variable investigated (pulse rate). On the other hand, the logistic regression analysis showed that the predictors for exaggerated increase in systolic blood pressure are old age and obesity. Physical therapy can be performed safely with the knowledge of the factors that may produce a serious increase in blood pressure during exercise therapy.

The following points limit the interpretation of our study results. First, the study sample $(n=23)$ was small, and therefore a definite conclusion cannot be drawn. Second, the present data were obtained in daily clinical practice, and therefore the exercise therapy was heterogeneous among patients and during the observation period in each patient. Third, medications, especially antihypertensive medication, may have affected the results. Moreover, the information about the mechanisms underlying the response of blood pressure cannot be verified from the results of the observational study. Despite these limitations, this pilot study provides useful information for physical therapists in daily clinical practice.

In conclusion, patients who experience a stroke or those who undergo orthopedic surgery may experience a significant increase in blood pressure as a response to exercise therapy for getting up. The increase in systolic blood pressure after 
exercise therapy is correlated with pulse rate at rest before the exercise therapy, and old age or increased body weight predicts exaggerated increase in blood pressure during exercise therapy.

\section{Funding}

This study was supported by Nagoya Gakuin University Grant (2018-2020).

\section{Conflict of interest}

The authors declare no conflict of interest.

\section{ACKNOWLEDGEMENT}

The authors would like to thank MARUZEN-YUSHODO Co. Ltd. (https://kw.maruzen.co.jp/kousei-honyaku/) for the English language editing.

\section{REFERENCES}

1) Umemura S, Arima H, Arima S, et al.: The Japanese Society of Hypertension Guidelines for the Management of Hypertension (JSH 2019). Hypertens Res, 2019, 42: 1235-1481. [Medline] [CrossRef]

2) Kaplan NM, Victor RG: Measurement of blood pressure. Kaplan's clinical hypertension, 11th ed. Philadelphia: Lippincott Williams \& Wilkins, 2015, pp 18-39.

3) Williams MA, Haskell WL, Ades PA, et al. American Heart Association Council on Clinical Cardiology, American Heart Association Council on Nutrition, Physical Activity, and Metabolism: Resistance exercise in individuals with and without cardiovascular disease: 2007 update: a scientific statement from the American Heart Association Council on Clinical Cardiology and Council on Nutrition, Physical Activity, and Metabolism. Circulation, 2007, 116: 572-584. [Medline] [CrossRef]

4) Miyamoto T, Kamada H, Moritani T: Acute cardiovascular responses to multiple sets of high-velocity resistance exercise in healthy adults. Res Sports Med, 2017, 25: 495-504. [Medline] [CrossRef]

5) Somani YB, Baross AW, Brook RD, et al.: Acute response to a 2-minute isometric exercise test predicts the blood pressure-lowering efficacy of isometric resistance training in young adults. Am J Hypertens, 2018, 31: 362-368. [Medline] [CrossRef]

6) Marques DL, Neiva HP, Faíl LB, et al.: Acute effects of low and high-volume resistance training on hemodynamic, metabolic and neuromuscular parameters in older adults. Exp Gerontol, 2019, 125: 110685. [Medline] [CrossRef]

7) Li Y, Thijs L, Hansen TW, et al. International Database on Ambulatory Blood Pressure Monitoring in Relation to Cardiovascular Outcomes Investigators: Prognostic value of the morning blood pressure surge in 5645 subjects from 8 populations. Hypertension, 2010, 55: 1040-1048. [Medline] [CrossRef]

8) Muller JE, Tofler GH, Stone PH: Circadian variation and triggers of onset of acute cardiovascular disease. Circulation, 1989, 79: 733-743. [Medline] [CrossRef]

9) Wielemborek-Musial K, Szmigielska K, Leszczynska J, et al.: Blood pressure response to submaximal exercise test in adults. BioMed Res Int, 2016, 2016: 5607507. [Medline] [CrossRef]

10) Albarrati AM: Outpatient physical therapy cardiovascular assessment: physical therapist perspective and experience. Physiother Theory Pract, 2019, 35: 843-850. [Medline] [CrossRef]

11) Anderson AD: The use of the heart rate as a monitoring device in an ambulation program: a progress report. Arch Phys Med Rehabil, 1964,45 : $140-146$. [Medline]

12) Seino Y, Nanjo K, Tajima N, et al. Committee of the Japan Diabetes Society on the Diagnostic Criteria of Diabetes Mellitus: Report of the committee on the classification and diagnostic criteria of diabetes mellitus. J Diabetes Investig, 2010, 1: 212-228. [Medline] [CrossRef]

13) Teramoto T, Sasaki J, Ueshima H, et al. Japan Atherosclerosis Society (JAS) Committee for Epidemiology and Clinical Management of Atherosclerosis: Diagnostic criteria for dyslipidemia. Executive summary of Japan Atherosclerosis Society (JAS) guideline for diagnosis and prevention of atherosclerotic cardiovascular diseases for Japanese. J Atheroscler Thromb, 2007, 14: 155-158. [Medline] [CrossRef]

14) Saccò M, Meschi M, Regolisti G, et al.: The relationship between blood pressure and pain. J Clin Hypertens (Greenwich), 2013, 15: 600-605. [Medline] [CrossRef]

15) O’Brien E, Pickering T, Asmar R, et al. Working Group on Blood Pressure Monitoring of the European Society of Hypertension: Working Group on Blood Pressure Monitoring of the European Society of Hypertension International Protocol for validation of blood pressure measuring devices in adults. Blood Press Monit, 2002, 7: 3-17. [Medline] [CrossRef]

16) Astrand I: A method for prediction of aerobic work capacity for females and males of different ages. Aerobic work capacity in men and women with special reference to age. Acta Physiol Scand, 1960, 49: 45-60. [CrossRef]

17) Fletcher GF, Balady GJ, Amsterdam EA, et al.: Exercise standards for testing and training: a statement for healthcare professionals from the American Heart Association. Circulation, 2001, 104: 1694-1740. [Medline] [CrossRef]

18) Mancia G, De Backer G, Dominiczak A, et al. The task force for the management of arterial hypertension of the European Society of Hypertension The task force for the management of arterial hypertension of the European Society of Cardiology: 2007 Guidelines for the management of arterial hypertension: The Task Force for the Management of Arterial Hypertension of the European Society of Hypertension (ESH) and of the European Society of Cardiology (ESC). Eur Heart J, 2007, 28: 1462-1536. [Medline]

19) Brett SE, Ritter JM, Chowienczyk PJ: Diastolic blood pressure changes during exercise positively correlate with serum cholesterol and insulin resistance. Circulation, 2000, 101: 611-615. [Medline] [CrossRef] 